\title{
Surveillance
}

\section{Mortalité associée à la maladie de Creutzfeldt-Jakob au Canada, de 1998 à 2013}

\author{
Coulthart $\mathrm{MB}^{1^{\star}}$, Jansen $\mathrm{GH}^{1,2}$, Connolly $\mathrm{T}^{1}$, $\mathrm{D}^{\prime}$ Amour $\mathrm{R}^{1}$, Kruse $\mathrm{J}^{1}$, Lynch $\mathrm{J}^{1}$, Sabourin $\mathrm{S}^{1}$, Wang $\mathrm{Z}^{1 \dagger}$, \\ Giulivi $\mathrm{A}^{2}$, Ricketts $\mathrm{MN}^{3}$, Cashman $\mathrm{NR}^{4}$
}

${ }^{1}$ Système canadien de surveillance de la maladie de Creutzfeldt-Jakob, Centre des maladies infectieuses d'origine alimentaire, environnementale et zoonotique, Agence de la santé publique du Canada, Ottawa (Ontario)

${ }_{3}^{2}$ Hôpital d'Ottawa, Université d'Ottawa et Association des laboratoires régionaux de l'Est de l'Ontario, Ottawa (Ontario)

${ }^{3}$ Université d'Ottawa, Ottawa (Ontario)

${ }^{4}$ Faculté de médecine (Neurologie), Centre de recherches sur le cerveau, Université de la Colombie-Britannique, Vancouver (Colombie-Britannique)

† Décédé en novembre 2014

*Correspondance : michael.coulthart@phac-aspc.gc.ca

Les auteurs dédient cet article à la mémoire de leur collègue et ami, Zheng Wang (1962-2014).

\section{Résumé}

Contexte : Les maladies humaines à prion, connues collectivement sous le nom de maladie de CreutzfeldtJakob $(\mathrm{MCJ})$, sont des maladies neurodégénératives infectieuses mortelles qui touchent toutes les populations humaines.

Objectif : Résumer les données nationales de surveillance de la maladie de Creutzfeldt-Jakob au Canada entre le $1^{\text {er }}$ janvier 1998 et le 31 décembre 2013.

Méthodologie : Des recherches approfondies ont été effectuées sur des cas individuels soupçonnés de maladie de Creutzfeldt-Jakob, en collaboration avec des professionnels de la santé et des chercheurs canadiens affiliés à un registre central de surveillance de la maladie de Creutzfeldt-Jakob exploité par l'Agence de la santé publique du Canada. Des données ont été recueillies sur le profil clinique, les antécédents familiaux et les résultats d'analyses paracliniques et de laboratoire, y compris d'une autopsie neuropathologique.

Résultats : Au total, 662 décès attribuables à une maladie de Creutzfeldt-Jakob certaine ou probable ont été recensés chez des résidents canadiens pendant la période à l'étude, dont 613 cas de maladie de CreutzfeldtJakob sporadique $(92,6 \%), 43$ cas de maladie génétique à prion $(6,5 \%), 4$ cas de maladie de CreutzfeldtJakob iatrogène $(0,6 \%)$ et 2 cas de variante de la maladie de Creutzfeldt-Jakob $(0,3 \%)$. Le taux de mortalité brut total de la maladie de Creutzfeldt-Jakob sporadique était de 1,18 par million par an [intervalle de confiance (IC) à $95 \%: 1,08 ; 1,27]$. Les taux selon l'âge étaient compris entre 0,05 [IC à $95 \%: 0,03 ; 0,08]$ chez les personnes de moins de 50 ans et 7,11 [IC à $95 \% ; 6,20 ; 8,11]$ chez les personnes âgées de 70 à 79 ans. Une tendance nette significative à la hausse des taux ajustés en fonction de l'âge a été observée au cours de la période à l'étude. Les indices comparatifs de mortalité, calculés individuellement pour dix provinces canadiennes par rapport aux taux de mortalité moyens nationaux, ne présentaient pas d'écart significatif par rapport à 1,0 .

Conclusion : La maladie de Creutzfeldt-Jakob reste rare au Canada, bien que les taux de mortalité varient de deux ordres de grandeur entre les groupes d'âge plus jeunes et plus âgés. La tendance à la hausse du taux de mortalité associé à la maladie de Creutzfeldt-Jakob sporadique normalisé en fonction de l'âge qui a été observée au cours de l'étude s'explique davantage par une amélioration progressive de la constatation des cas que par une augmentation réelle de l'incidence de la maladie. 


\section{Introduction}

Les maladies à prion sont des maladies dégénératives du cerveau rares et mortelles qui touchent les humains et plusieurs autres mammifères. Fait remarquable, ces maladies se transmettent par un processus moléculaire ressemblant à une infection, impliquant une forme pathologiquement modifiée, qui se propage spontanément, d'une glycoprotéine hôte, la protéine prion (PrP). Les agents infectieux de ces maladies sont par conséquent appelés prions, ou particules protéiques infectieuses (1). Malgré les similitudes récemment reconnues entre les mécanismes moléculaires des maladies à prion associées au comportement pathologique de la PrP et les autres maladies de type maladie à prion dues à un repliement anormal d'autres protéines, il n'existe à ce jour aucune preuve que les maladies de type maladie à prion sont transmissibles entre les individus dans des conditions naturelles $(2,3)$. Les maladies à prion présentent donc une combinaison unique de difficultés pour la santé publique, notamment des périodes d'incubation longues et silencieuses, l'absence d'immunité protectrice de l'hôte, la résistance à la décontamination, les outils de diagnostic limités et le risque de transmission zoonotique et par les soins de santé (4).

Les maladies à prion des humains - par souci de concision, désignées ici collectivement sous le nom de maladie de Creutzfeldt-Jakob - présentent diverses manifestations clinicopathologiques qui reflètent des origines étiologiques endogènes et exogènes distinctes. Parmi les formes « classiques » de la maladie de Creutzfeldt-Jakob, le sous-type le plus courant, la maladie de Creutzfeldt-Jakob sporadique (MCJs), se définit par une neuropathologie caractéristique du prion (en particulier la dégénérescence spongiforme et le dépôt de PrP pathologique dans les tissus cérébraux) en l'absence de cause génétique ou infectieuse apparente. La maladie de Creutzfeldt-Jakob sporadique semble toucher de façon endémique toutes les populations, avec un taux de mortalité moyen de un à deux cas par million par an, et constitue généralement $85 \%$ à $95 \%$ de l'ensemble des cas de maladie de Creutzfeldt-Jakob recensés au moyen de la surveillance épidémiologique (5). Les formes de la maladie d'origine génétique (5\% à $15 \%$ des cas de maladie de Creutzfeldt-Jakob classique), qui semblent également se produire universellement, sont associées à l'une des plus de 50 mutations pathogènes différentes du gène hôte qui encode la PrP. Certaines de ces formes génétiques - en particulier le syndrome de Gerstmann-Straüssler-Scheinker et l'insomnie fatale familiale - présentent des caractéristiques clinicopathologiques distinctives et peuvent généralement être diagnostiquées comme étant d'origine génétique même si la mutation génétique sous-jacente spécifique est inconnue (6).

Des cas iatrogènes de maladie de Creutzfeldt-Jakob classique (MCJi) se sont produits par transmission accidentelle du prion en milieu de soins de santé, en particulier lors de l'utilisation thérapeutique de tissus ou d'extraits tissulaires cadavériques vraisemblablement contaminés provenant de dons de personnes atteintes d'une maladie de Creutzfeldt-Jakob sporadique non reconnue. Bien que la MCJi soit très rare, les épidémies ont été très bien documentées, avec 469 cas signalés dans le monde entier jusqu'en 2012 (7). De plus, des études épidémiologiques soigneusement menées ont mis au jour de nouvelles preuves démontrant qu'une partie des cas de maladie de Creutzfeldt-Jakob « sporadique » peut être attribuable à une exposition iatrogène éloignée lors d'une intervention chirurgicale, en particulier au début de la vie $(8,9)$. Plus récemment, une nouvelle maladie à prion humaine, la variante de la maladie de Creutzfeldt-Jakob (vMCJ), est apparue au milieu des années 1990 au Royaume-Uni par transmission zoonotique aux humains d'une maladie à prion des bovins, l'encéphalopathie spongiforme bovine (ESB) (10-12). En date d'avril 2015, 229 cas de vMCJ ont été signalés chez des résidents de 12 pays, dont deux cas importés au Canada $(13,14)$. Parmi ce nombre figurent cinq cas probables, tous recensés au Royaume-Uni, de transmission secondaire (d'humain à humain) par le sang de l'infection par la vMCJ (15-17).

Poussés par le besoin de mieux comprendre l'épidémiologie de la maladie de Creutzfeldt-Jakob, ainsi que par la responsabilité de faire face rapidement et habilement aux risques de transmission iatrogène et zoonotique, des systèmes spécialisés dans la surveillance épidémiologique prospective de la maladie de Creutzfeldt-Jakob dans la population ont commencé à se mettre en place dans de nombreux pays au début des années 1990 (18). Au Canada, la motivation initiale est venue d'une question soulevée par la Commission d'enquête sur l'approvisionnement en sang au Canada (Commission Krever), concernant la possibilité que la maladie de Creutzfeldt-Jakob se transmette par le sang $(19,20)$. En réponse à cette question, en avril 1998, le 
gouvernement du Canada a officiellement lancé la surveillance épidémiologique nationale prospective de la maladie de Creutzfeldt-Jakob par le biais du Système canadien de surveillance de la maladie de CreutzfeldtJakob (SS-MCJ), aujourd'hui exploité par l'Agence de la santé publique du Canada (21). Depuis 2000, toutes les formes de la maladie de Creutzfeldt-Jakob sont des maladies à déclaration obligatoire à l'échelle nationale au Canada, et elles le sont désormais dans toutes les provinces et tous les territoires. Le présent article décrit un aperçu des observations de ce programme de surveillance nationale sur une période de 16 ans, allant du $1^{\text {er }}$ janvier 1998 au 31 décembre 2013.

\section{Méthodologie}

\section{Collecte des données}

Le SS-MCJ emploie une méthodologie établie à l'échelle internationale pour la surveillance épidémiologique de la maladie de Creutzfeldt-Jakob (18). Conformément à cette méthodologie, après notification par un professionnel de la santé d'un cas soupçonné de maladie de Creutzfeldt-Jakob chez un résident canadien, les chercheurs du SS-MCJ collaborent pour faciliter les examens diagnostiques, constituer et organiser les dossiers de cas, interpréter et diffuser les résultats, aider à la déclaration de la maladie et analyser et gérer les problèmes de santé publique. Le SS-MCJ utilise le registre central des cas et une base de données électronique pour suivre les événements, les taux et les tendances de l'incidence de la maladie de CreutzfeldtJakob au Canada. Les professionnels de la santé collaborateurs, ainsi que le public, peuvent accéder plus facilement aux chercheurs du SS-MCJ grâce à une ligne téléphonique sans frais (1-888-489-2999) et à une adresse de courriel dédiée (cjdss@phac-aspc.gc.ca).

\section{Définitions de cas}

L'examen diagnostique de la maladie de Creutzfeldt-Jakob et des autres encéphalopathies subaiguës chez le patient vivant est souvent difficile (22). C'est pourquoi une surveillance épidémiologique d'excellente qualité suppose de recueillir divers types de données justificatives, ainsi que d'effectuer un suivi des cas individuels en temps réel. Étant donné qu'un diagnostic de maladie de Creutzfeldt-Jakob certaine nécessite un examen neuropathologique, l'autopsie crânienne est systématiquement envisagée. Les cas pour lesquels l'examen neuropathologique n'a pas été effectué peuvent être classés comme des cas de maladie de Creutzfeldt-Jakob probable sur la base de critères non neuropathologiques, tels que le profil clinique, les résultats des immunoessais sur les protéines du liquide céphalorachidien, les observations à l'aide de l'électroencéphalographie ou de l'imagerie par résonance magnétique, et l'analyse génétique. Lorsque l'information est encore plus limitée, il peut être envisageable d'attribuer au cas le plus faible degré de classification diagnostique formelle, à savoir celui de maladie de Creutzfeldt-Jakob possible. Les définitions des cas de surveillance employées par le SS-MCJ, qui ont été publiées par le Système national de surveillance des maladies à déclaration obligatoire de l'Agence de la santé publique du Canada, sont fondées sur les définitions utilisées à l'échelle internationale $(18,23)$.

\section{Calculs des taux de mortalité}

Bien que la surveillance nationale prospective de la maladie de Creutzfeldt-Jakob au Canada ait officiellement débuté le $1^{\text {er }}$ avril 1998, des données existaient également sur les décès attribuables à la maladie de Creutzfeldt-Jakob survenus en janvier, février et mars 1998. Par conséquent, les données présentées dans ce rapport incluent les décès attribuables à la maladie de Creutzfeldt-Jakob au Canada recensés par le SS-MCJ comme étant survenus entre le $1^{\text {er }}$ janvier 1998 et le 31 décembre 2013. Compte tenu de la portée nationale du SS-MCJ et de son approche basée sur la population, aux fins du présent rapport, la population entière du Canada a été utilisée pour estimer les taux de mortalité, qui sont exprimés ici en cas unitaires par million d'habitants par année. II convient de noter également que, bien qu'aucun cas de maladie de Creutzfeldt-Jakob n'ait été recensé dans les territoires canadiens (Yukon, Territoires du Nord-Ouest et Nunavut) pendant la période de surveillance, étant donné que le SS-MCJ était prêt à accepter des déclarations de cas de ces régions, leurs populations ont été incluses dans les dénominateurs des estimations du taux national. Les chiffres de la population canadienne et les données démographiques ont été adaptés à partir de la base de données socioéconomiques en ligne CANSIM de Statistique Canada (24). Sur la base de ces données, pour 
l'ensemble de la période à l'étude, la surveillance a porté sur un total nominal de 521794297 annéespersonnes, où une année-personne représente l'appartenance d'une personne à une population vulnérable sur une période d'une année (25).

Les intervalles de confiance (IC) à $95 \%$ exacts pour les estimations du taux de mortalité ont été dérivés à partir d'un modèle de Poisson des nombres de cas. L'hypothèse d'une tendance linéaire des taux de mortalité ajustés en fonction de l'âge pendant la période de surveillance a été testée au moyen d'une régression linéaire non paramétrique, dans laquelle la pente de la régression a été calculée en prenant la médiane des pentes reliant toutes les paires de points de données. De plus, un test bilatéral de la corrélation des rangs entre l'année et le taux de mortalité a été effectué au moyen du coefficient tau de Kendall, qui est basé sur la proportion de paires d'observations de deux variables dans lesquelles les rangs respectifs des deux observations sont concordants (26). Des indices comparatifs de mortalité (ICM) pour la MCJs (définis comme le rapport entre les taux de mortalité totaux observés dans une population à l'étude et les taux de mortalité « attendus » dans la population à l'étude dérivés de taux selon l'âge connus pour une population de référence) ont été estimés pour chaque province. Les taux de mortalité nationaux de la maladie de Creutzfeldt-Jakob ont été utilisés pour calculer les taux attendus, en basant ici encore les IC à 95 \% sur un modèle de Poisson.

Compte tenu des faibles nombres de cas recensés de maladie génétique à prion, de MCJi et de vMCJ (voir cidessous), seule une analyse statistique limitée a été effectuée pour ces sous-types de la maladie, et les résultats présentés ici consistent principalement en des nombres de cas et des estimations des taux de mortalité bruts pour la maladie génétique à prion.

\section{Cadre éthique}

L'inscription formelle d'un patient auprès du SS-MCJ (recommandation de cas) est effectuée avec un consentement éclairé par écrit pour chaque volet principal de la collecte de données (autopsie, génétique, consultation du dossier médical et entretien) selon un protocole approuvé par le Comité d'éthique de la recherche de Santé Canada et l'Agence de la santé publique du Canada (Certificat REB-2009-0036). Les données personnelles recueillies sont enregistrées auprès du gouvernement du Canada sous forme d'un fichier de renseignements personnels (27).

\section{Résultats}

\section{Rendement diagnostique et qualité}

Entre le $1^{\mathrm{er}}$ janvier 1998 et le 31 décembre 2013, le SS-MCJ a ouvert 1420 dossiers de recommandations de cas sur des patients soupçonnés d'être atteints de la maladie de Creutzfeldt-Jakob ou risquant de l'être (c.-à-d. membres de la famille de patients atteints de maladie génétique à prion). Au total, 3689 essais en laboratoire ont été effectués par les laboratoires de l'Agence de la santé publique du Canada, comprenant 2193 immunoessais sur les protéines du liquide céphalorachidien, 635 analyses génétiques et 861 examens neuropathologiques, dont 828 autopsies crâniennes complètes (Tableau 1). Au cours de la même période, les décès de 662 résidents canadiens ont été attribués à une maladie de Creutzfeldt-Jakob certaine ou probable, parmi lesquels figuraient 613 cas de MCJs (92,6 \%), 43 cas de maladie génétique à prion (6,5\%), 4 cas de MCJi $(0,6 \%)$ et 2 cas de vMCJ $(0,3 \%)$. Parmi les 662 diagnostics de maladie de Creutzfeldt-Jakob, 535 $(80,8 \%)$ étaient certains et $127(19,2 \%)$ étaient probables. En outre, 22 décès ont été attribués à une maladie de Creutzfeldt-Jakob possible, et 77 cas étaient inclassables. Sur les 613 diagnostics de MCJs, 498 (81,2 \%) étaient certains et $115(18,8 \%)$ étaient probables. Parmi 575 diagnostics de non-maladie de Creutzfeldt-Jakob, cette dernière a été exclue par neuropathologie dans 308 cas $(53,6 \%)$ et par d'autres critères dans 267 cas $(46,4 \%)$. 
Tableau 1 : Essais en laboratoire, recommandations de cas et décès attribués à la maladie de Creutzfeldt-Jakob au Canada, de 1998 à 2013

\begin{tabular}{|c|c|c|c|c|c|c|}
\hline Année $^{1}$ & Essais $^{2}$ & Recommandations ${ }^{3,4}$ & Autopsies $^{5}$ & Décès $^{6}$ & Certaine $^{7}$ & Probable $^{8}$ \\
\hline 1998 & 50 & 44 & 24 & 24 & 20 & 4 \\
\hline 1999 & 84 & 63 & 34 & 32 & 28 & 4 \\
\hline 2000 & 126 & 82 & 42 & 35 & 32 & 3 \\
\hline 2001 & 161 & 101 & 49 & 30 & 23 & 7 \\
\hline 2002 & 158 & 103 & 54 & 36 & 32 & 4 \\
\hline 2003 & 137 & 75 & 44 & 29 & 27 & 2 \\
\hline 2004 & 268 & 90 & 63 & 44 & 33 & 11 \\
\hline 2005 & 257 & 97 & 58 & 44 & 37 & 7 \\
\hline 2006 & 234 & 80 & 57 & 44 & 37 & 7 \\
\hline 2007 & 259 & 101 & 49 & 39 & 33 & 6 \\
\hline 2008 & 305 & 100 & 57 & 49 & 34 & 15 \\
\hline 2009 & 346 & 104 & 74 & 53 & 46 & 7 \\
\hline 2010 & 281 & 76 & 46 & 38 & 31 & 7 \\
\hline 2011 & 342 & 102 & 54 & 51 & 40 & 11 \\
\hline 2012 & 351 & 103 & 69 & 63 & 46 & 17 \\
\hline 2013 & 330 & 99 & 54 & 51 & 36 & 15 \\
\hline Total & 3689 & 1420 & 828 & 662 & 535 & 127 \\
\hline
\end{tabular}

${ }^{1}$ Du $1^{\mathrm{er}}$ janvier au 31 décembre de chaque année indiquée.

${ }^{2}$ Tous les essais en laboratoire associés à la maladie de Creutzfeldt-Jakob, y compris les analyses des marqueurs protéiques dans le liquide céphalorachidien, les analyses génétiques et les examens neuropathologiques.

${ }^{3}$ Une recommandation est définie comme l'inscription d'un patient auprès du SS-MCJ.

${ }^{4}$ À noter que les résultats de certaines recommandations n'avaient pas été déterminés à la fin de l'année 2013.

${ }^{5}$ Une autopsie est définie comme une autopsie crânienne complète.

${ }^{6}$ Décès attribuables à une maladie de Creutzfeldt-Jakob certaine ou probable.

${ }^{7}$ Diagnostic confirmé par neuropathologie.

${ }^{8}$ Diagnostic étayé par des critères non neuropathologiques.

\section{Répartition de la MCJs selon l'âge et le sexe}

La Figure 1 illustre la répartition des âges au moment du décès pour les 613 cas de MCJs. La répartition présentait un écart significatif par rapport à la normalité, dû à une asymétrie vers la gauche (coefficient de D'Agostino-Pearson : -0,297; $p=0,012$ ). L'âge au moment du décès était compris entre 33 et 92 ans, avec un âge médian de 69 ans [IC à $95 \%: 68 ; 70$ ] et un écart interquartile de 62 à 75 ans. Les hommes étaient au nombre de 291 (47,5\%) [IC à $95 \%: 43,5 ; 51,4]$ et les femmes au nombre de $322(52,4 \%)$ [IC à $95 \%: 48,6$; $56,5]$, ce qui indique un rapport de masculinité impossible à distinguer de 1 . Les répartitions selon l'âge étaient également très similaires entre les sexes, avec un âge médian de 68 ans chez les hommes [IC à $95 \%: 67 ; 70$; écart interquartile : 60;74;75] et un âge médian de 69 ans chez les femmes [IC à $95 \%$ : 67; 70; écart interquartile : 61;75]. 
Figure 1 : Répartition selon l'âge au moment du décès de 613 cas de maladie de Creutzfeldt-Jakob sporadique au Canada, de 1998 à $2013^{1-3}$



\footnotetext{
${ }^{1}$ Les décès des hommes et des femmes sont regroupés dans le tracé.

${ }^{2}$ Les âges sont groupés en tranches de cinq années.

${ }^{3}$ La courbe normale est tracée (ligne pointillée) selon la moyenne $(67,8)$ et l'écart-type $(9,4)$ de l'échantillon.
}

\section{Mortalité associée à la MCJs}

Le Tableau 2 présente les nombres de cas et les taux de mortalité en fonction de l'âge associés à la MCJs pour cinq tranches d'âge : $\leq 49$ ans, 50 à 59 ans, 60 à 69 ans, 70 à 79 ans, et $\geq 80$ ans. Les taux bruts variaient fortement en fonction de l'âge, allant de 0,05 pour la tranche d'âge de $\leq 49$ ans à 7,11 pour la tranche d'âge de 70 à 79 ans; parmi les 613 cas de MCJs, seulement 17 (2,8\%) ont été recensés chez des personnes âgées de $\leq 49$ ans. Le taux de mortalité brut total, toutes tranches d'âge confondues, sur l'ensemble de la période de surveillance était de 1,18, avec des taux annuels compris entre 0,73 en 1998 et 1,78 en 2012. Les taux normalisés en fonction de l'âge ont également été calculés, en prenant le recensement de la population canadienne de 2006 comme référence (Tableau 2). Ces taux allaient de 0,84 en 1998 à 1,65 en 2012, pour un taux total sur la période de surveillance de 1,20. Lorsqu'ils ont été soumis à une analyse par régression linéaire non paramétrique, les taux normalisés en fonction de l'âge étaient significativement associés à l'année, selon la fonction linéaire : taux =0,029 (année) - 57,30 [IC à $95 \%: 0,002 ; 0,052$ pour l'estimation de la pente] (Figure 2). Le coefficient tau de Kendall de corrélation des rangs $(0,45)$ était significatif à $p=0,017$ (test bilatéral).

Tableau 2 : Taux de mortalité en fonction de l'âge et total annuel associé à la maladie de Creutzfeldt-Jakob sporadique au Canada, de 1998 à 2013

\begin{tabular}{|c|c|c|c|c|c|c|c|c|}
\hline \multirow[b]{2}{*}{ Année $^{1}$} & \multicolumn{6}{|c|}{$\hat{A} g e^{2}$} & \multicolumn{2}{|c|}{ Mortalité $^{3}$} \\
\hline & $\leq 49$ & 50 à 59 & 60 à 69 & 70 à 79 & $\geq 80$ & $\begin{array}{l}\text { Tous âges } \\
\text { confondus }\end{array}$ & Brute $^{4}$ & $\begin{array}{c}\text { Ajustée selon } \\
\text { l'âge }^{5}\end{array}$ \\
\hline 1998 & 0 & 3 & 5 & 12 & 2 & 22 & $0,73[0,46 ; 1,10]$ & $0,84[0,52 ; 1,27]$ \\
\hline 1999 & 2 & 4 & 9 & 10 & 2 & 27 & $0,89[0,58 ; 1,29]$ & $0,96[0,63 ; 1,39]$ \\
\hline 2000 & 1 & 4 & 5 & 14 & 8 & 32 & $1,04[0,71 ; 1,47]$ & $1,23[0,84 ; 1,75]$ \\
\hline 2001 & 0 & 4 & 12 & 8 & 3 & 27 & $0,87[0,57 ; 1,26]$ & $0,98[0,64 ; 1,42]$ \\
\hline
\end{tabular}




\begin{tabular}{|c|c|c|c|c|c|c|c|c|}
\hline $\mathbf{2 0 0 2}$ & 2 & 5 & 6 & 14 & 4 & 31 & $0,99[0,67 ; 1,40]$ & $1,07[0,73 ; 1,52]$ \\
\hline $\mathbf{2 0 0 3}$ & 2 & 1 & 13 & 11 & 0 & 27 & $0,85[0,56 ; 1,24]$ & $0,91[0,60 ; 1,32]$ \\
\hline $\mathbf{2 0 0 4}$ & 0 & 10 & 14 & 13 & 5 & 42 & $1,31[0,95 ; 1,77]$ & $1,40[1,01 ; 1,90]$ \\
\hline $\mathbf{2 0 0 5}$ & 1 & 8 & 12 & 21 & 0 & 42 & $1,30[0,94 ; 1,76]$ & $1,36[0,98 ; 1,83]$ \\
\hline $\mathbf{2 0 0 6}$ & 0 & 5 & 13 & 17 & 4 & 39 & $1,19[0,85 ; 1,63]$ & $1,24[0,88 ; 1,69]$ \\
\hline $\mathbf{2 0 0 7}$ & 2 & 7 & 14 & 8 & 4 & 35 & $1,06[0,74 ; 1,48]$ & $1,07[0,75 ; 1,49]$ \\
\hline $\mathbf{2 0 0 8}$ & 0 & 9 & 13 & 18 & 8 & 48 & $1,44[1,06 ; 1,91]$ & $1,44[1,06 ; 1,91]$ \\
\hline $\mathbf{2 0 0 9}$ & 1 & 7 & 19 & 16 & 5 & 48 & $1,42[1,05 ; 1,89]$ & $1,39[1,02 ; 1,84]$ \\
\hline $\mathbf{2 0 1 0}$ & 2 & 6 & 19 & 4 & 4 & 35 & $1,03[0,71 ; 1,43]$ & $0,96[0,67 ; 1,33]$ \\
\hline $\mathbf{2 0 1 1}$ & 1 & 8 & 20 & 12 & 5 & 46 & $1,33[0,98 ; 1,78]$ & $1,24[0,91 ; 1,66]$ \\
\hline $\mathbf{2 0 1 2}$ & 1 & 13 & 22 & 20 & 6 & 62 & $1,78[1,36 ; 2,28]$ & $1,65[1,26 ; 2,11]$ \\
\hline $\mathbf{2 0 1 3}$ & 2 & 4 & 19 & 22 & 3 & 50 & $1,42[1,05 ; 1,87]$ & $1,30[0,96 ; 1,71]$ \\
\hline $\begin{array}{c}\text { Toutes } \\
\text { années } \\
\text { confondues }\end{array}$ & 17 & 98 & 215 & 220 & 63 & 613 & $1,18[1,08 ; 1,27]$ & $1,20[1,11 ; 1,30]$ \\
\hline $\begin{array}{c}\text { Mortalité } \\
\text { Mort }\end{array}$ & $\begin{array}{c}0,05 \\
{[0,03 ; 0,08]}\end{array}$ & $\begin{array}{c}1,41 \\
{[1,14 ; 1,72]}\end{array}$ & $\begin{array}{c}4,59 \\
{[4,00 ; 5,25]}\end{array}$ & $\begin{array}{c}7,11 \\
{[6,20 ; 8,11]}\end{array}$ & $\begin{array}{c}3,44 \\
{[2,64 ; 4,40]}\end{array}$ & $\begin{array}{c}1,18 \\
{[1,08 ; 1,27]}\end{array}$ & - & - \\
\hline
\end{tabular}

${ }^{1}$ Les intervalles de temps vont du $1^{\mathrm{er}}$ janvier au 31 décembre de chaque année civile.

${ }^{2}$ L'âge est exprimé en nombre d'années de vie révolues au moment du décès.

${ }^{3}$ Les taux de mortalité sont exprimés en nombre de décès par million d'habitants par année, avec un intervalle de confiance à $95 \%$ (entre crochets) basé sur un modèle de Poisson.

${ }^{4}$ D'après les estimations de Statistique Canada de la population canadienne totale au quatrième trimestre de chaque année.

${ }^{5}$ D'après le recensement canadien de 2006.

Figure 2 : Taux de mortalité normalisé en fonction de l'âge associé à la maladie de Creutzfeldt-Jakob sporadique au Canada, de 1998 à $2013^{1-3}$



\footnotetext{
${ }^{1}$ Les taux de mortalité sont exprimés par million d'habitants par année.

${ }^{2}$ Pour la normalisation directe, la population du recensement canadien de 2006 a été utilisée comme population de référence.

${ }^{3}$ Pente de régression linéaire non paramétrique (ligne pointillée) = médiane des pentes entre toutes les paires de données.
} 
Pour étudier la possibilité d'une variation géographique des taux de mortalité associés à la maladie de Creutzfeldt-Jakob pendant la période à l'étude, les indices comparatifs de mortalité (ICM) et les IC à $95 \%$ ont été estimés pour chaque province, en prenant les taux de mortalité nationaux en fonction de l'âge estimés comme référence pour calculer les taux attendus. Les résultats sont présentés dans la Figure 3. On peut constater que les estimations des indices comparatifs de mortalité et les amplitudes de leurs IC à $95 \%$ respectifs variaient considérablement, l'écart de ces derniers étant prévisible compte tenu de la variation des nombres de cas entre les provinces peu ou très peuplées (fourchette : $<5$ cas [île-du-Prince-Édouard] à 209 cas [Ontario]). Malgré cette variation, les IC à $95 \%$ des indices comparatifs de mortalité pour toutes les provinces incluaient la valeur 1,0, ce qui permet de déduire qu'il n'existe pas de données statistiques pour étayer une variation géographique des taux de mortalité.

Figure 3 : Indices comparatifs de mortalité pour la maladie de Creutzfeldt-Jakob dans les provinces canadiennes, de 1998 à $2013^{1-4}$

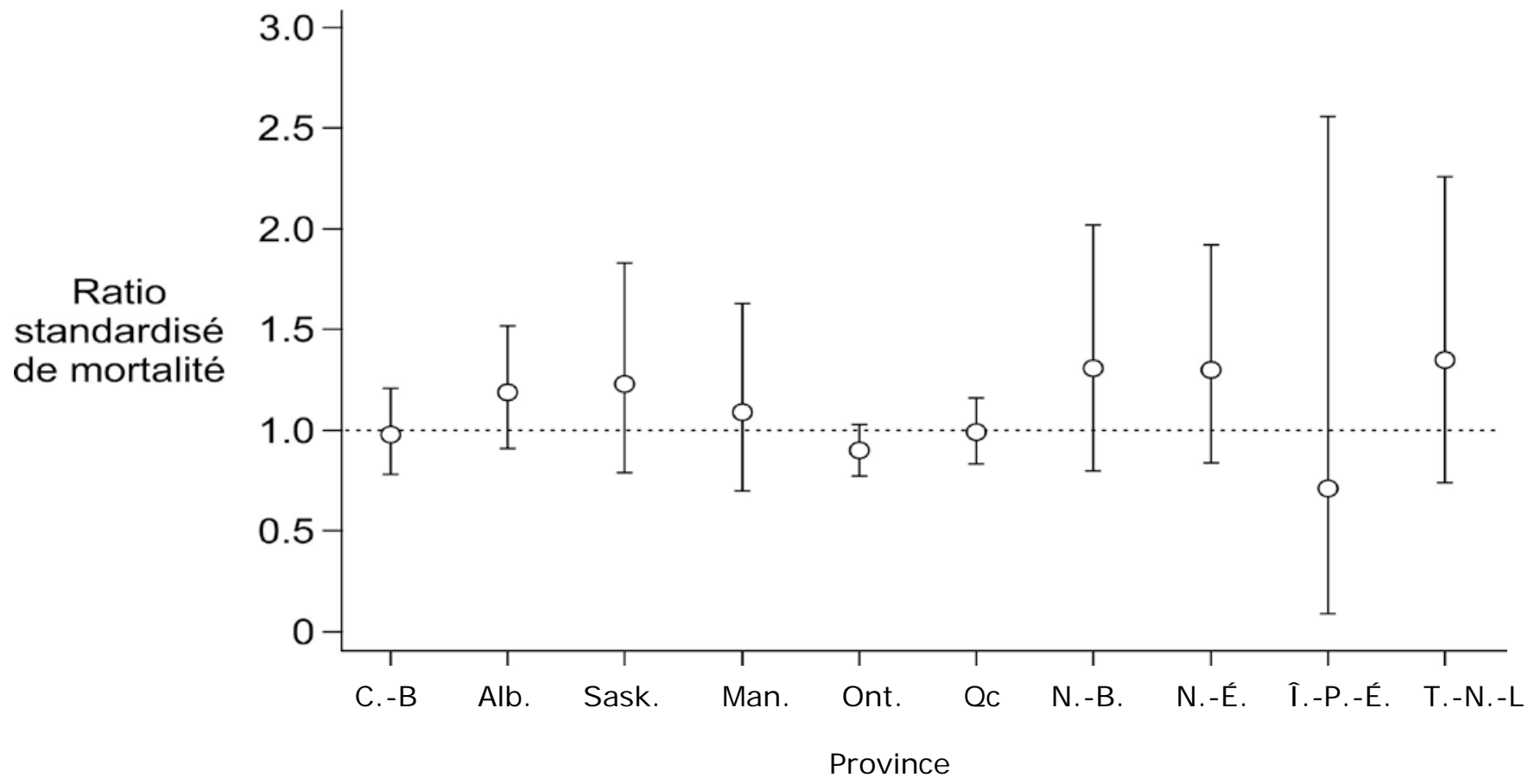

\footnotetext{
${ }^{1}$ Abréviations des noms des provinces : C.-B. - Colombie-Britannique; Alb. - Alberta; Sask. - Saskatchewan; Man. - Manitoba; Ont. - Ontario; Qc - Québec; N.-B. - Nouveau-Brunswick; N.-É. - Nouvelle-Écosse; Î.-P.-É. - Île-du-Prince-Édouard; T.-N.-L - Terre-Neuve-et-Labrador.

${ }^{2}$ Les symboles ouverts représentent les estimations ponctuelles des indices comparatifs de mortalité (ICM).

${ }^{3}$ Les barres verticales représentent les intervalles de confiance à $95 \%$ des indices comparatifs de mortalité, basés sur un modèle de Poisson.

${ }^{4}$ Les indices comparatifs de mortalité ont été calculés en prenant le taux de mortalité moyen national associé à la MCJs comme taux de référence.
}

\section{Maladie génétique à prion, MCJi et vMCJ}

Les caractéristiques de base des 43 cas recensés de maladie génétique à prion sont résumées dans le Tableau 3. Des cas des trois présentations clinicopathologiques classiques connues de la maladie génétique à prion (maladie de Creutzfeldt-Jakob, syndrome de Gerstmann-Sträussler-Scheinker, insomnie fatale familiale) ont été recensés. Les taux d'autopsie étaient également élevés dans ce groupe, avec 33 diagnostics sur 43 (77\%) étayés par neuropathologie en plus d'une mutation identifiée du gène hôte qui encode la PrP. II est intéressant de noter que, sur les 20 cas de syndrome de Gerstmann-Sträussler-Scheinker, 9 (45\%) n'ont été découverts qu'au moment de l'autopsie et ne présentaient pas de mutation identifiée, car l'analyse génétique n'avait pas été demandée. II apparaît clairement que le taux de mortalité total associé aux maladies génétiques 
à prion pendant la période à l'étude était nettement inférieur à celui associé à la $M C J s$, avec 0,08 décès par million par an [IC à $95 \%: 0,06 ; 0,11]$. Enfin, quatre cas de MCJi (présentant tous une greffe de dure-mère comme facteur de risque) et deux cas de vMCJ (un cas certain et un cas probable) ont été recensés.

Tableau 3 : Caractéristiques des 43 cas de maladie génétique à prion recensés au Canada, de 1998 à 2013

\begin{tabular}{|c|c|c|c|c|c|}
\hline Sous-type $^{1}$ & $\begin{array}{l}\text { Mutation } \\
\text { identifiée }^{2}\end{array}$ & $\begin{array}{c}\text { Mutation non } \\
\text { identifiée }^{3}\end{array}$ & $\begin{array}{c}\text { Certaine } \\
(\%)^{4}\end{array}$ & Probable $(\%)^{5}$ & Total \\
\hline MCJ & 21 & 0 & $16(76)$ & $5(24)$ & 21 \\
\hline GSS & 11 & 9 & $16(80)$ & $4(20)$ & 20 \\
\hline IFF & 2 & 0 & $1(50)$ & $1(50)$ & 2 \\
\hline Tous & 34 & 9 & $33(79)$ & $10(21)$ & 43 \\
\hline
\end{tabular}

${ }^{1}$ MCJ - maladie de Creutzfeldt-Jakob; GSS - syndrome de Gerstmann-Sträussler-Scheinker; IFF - insomnie fatale familiale.

${ }^{2}$ Modification de l'ADN étiologique identifiée.

${ }^{3}$ Modification de l'ADN étiologique non identifiée (aucune analyse génétique effectuée).

${ }^{4}$ Diagnostic confirmé par neuropathologie.

${ }^{5}$ Diagnostic étayé par des critères non neuropathologiques.

\section{Discussion}

D'après les données de la surveillance épidémiologique prospective nationale, au total, 662 décès ont été attribués à toutes les formes de maladie de Creutzfeldt-Jakob au Canada entre 1998 et 2013. En ce qui concerne le sous-type étiologique le plus courant, la MCJs (92,6 \% de tous les cas), nous avons estimé un taux de mortalité brut moyen total de 1,18 décès certain ou probable par million par an. Nous avons également confirmé la forte corrélation entre les taux de mortalité bruts associés à la MCJs et l'âge, avec un taux selon l'âge très faible de 0,05 chez les personnes de moins de 50 ans et un taux maximum de 7,11 dans la tranche d'âge de 70 à 79 ans. Nos données ont aussi permis de confirmer un autre schéma épidémiologique connu, à savoir qu'aucun biais lié au sexe n'était détectable dans les taux de mortalité. Nous avons mis au jour des données probantes à l'appui d'une modeste tendance nette à la hausse du taux de mortalité normalisé en fonction de l'âge associé à la MCJs sur la période de 16 ans. Toutefois, en accord avec la forte couverture nationale, aucune justification statistique n'a été trouvée pour étayer un écart géographique du taux par rapport au taux attendu, estimé à partir du taux national, dans aucune des dix provinces canadiennes où des cas ont été observés.

L'épidémiologie de la maladie de Creutzfeldt-Jakob a été étudiée systématiquement pendant près de quatre décennies (5-7,12,28-30). Les premières estimations de la mortalité, fondées principalement sur des approches rétrospectives de l'identification des cas, étaient en général nettement inférieures à un cas par million par an (29). Une approche prospective plus structurée de la recherche des cas appliquée en France entre 1978 et 1982 a fourni la première démonstration bien contrôlée d'un gradient des taux observés à l'intérieur d'un pays (0,56 pour la France, 0,86 pour l'agglomération de Paris et 1,19 pour la ville de Paris), qui représente une corrélation positive des taux avec le degré d'urbanisation et semble indiquer un effet de constatation significatif (30). La combinaison des études rétrospectives et prospectives menées en France et, par la suite, au Royaume-Uni a également démontré une hausse de la mortalité apparente avec le temps, laquelle a aussi été attribuée à une amélioration de la constatation des cas $(30,31)$.

Les estimations plus récentes des taux de mortalité associés à la maladie de Creutzfeldt-Jakob tendent vers une fourchette de un à deux cas par million par an (5). Cette évolution s'est produite parallèlement à des améliorations méthodologiques, telles que l'établissement de registres nationaux, l'application d'une approche prospective, la formulation de définitions normalisées des cas, la reconnaissance des sous-types de la maladie, l'élaboration et la validation d'outils de diagnostic, et la prise de conscience accrue parmi les professionnels de la santé collaborateurs, qui renforcent toutes la plausibilité d'une amélioration de la constatation des cas, en particulier chez les patients âgés et/ou chez les patients présentant des manifestations moins typiques $(5,32)$. En Suisse, une hausse temporaire de la mortalité associée à la maladie de Creutzfeldt-Jakob entre 2001 et 
2004 a finalement été attribuée à une sensibilisation accrue à la maladie à la suite de l'épidémie de vMCJ au Royaume-Uni; les auteurs sont même allés jusqu'à suggérer que le « véritable » taux de mortalité de la MCJs pouvait dépasser deux cas par million par an (33). Dans une étude récente intégrant des données de dix pays, des corrélations statistiques significatives ont été démontrées entre le taux de mortalité observé associé à la MCJs et plusieurs indices d'intensité de la surveillance, notamment les taux de déclaration de cas au registre central ainsi que les taux d'autopsie ou d'autres analyses en laboratoire (34). À la lumière de ces considérations, nous sommes d'avis que la tendance à la hausse observée des taux de mortalité associés à la MCJs normalisés en fonction de l'âge au Canada entre 1998 et 2013 peut s'expliquer davantage par une amélioration progressive de la constatation des cas que par des causes intrinsèques entraînant une réelle augmentation du taux. Par conséquent, bien que la sous-constatation puisse légitimement être considérée comme une limitation potentielle de la présente étude, une telle tendance positive laisse à penser que des améliorations plus poussées pourraient être réalisées. Compte tenu de la forte corrélation entre les taux de mortalité et l'âge, il est également utile de garder à l'esprit la possibilité que le vieillissement démographique contribue à de véritables augmentations des taux de MCJs à l'avenir.

Les observations tirées de la surveillance qui sont présentées ici confirment et vont au-delà de celles d'études publiées auparavant qui comprenaient des données rétrospectives ou prospectives sur la mortalité associée à la maladie de Creutzfeldt-Jakob au Canada, et sont cohérentes avec celles d'autres systèmes de surveillance de la maladie de Creutzfeldt-Jakob à l'échelle internationale (5,34-36). En plus de fournir des estimations d'excellente qualité des taux de mortalité totaux, en fonction de l'âge et en fonction du sexe pour les formes classiques de la maladie de Creutzfeldt-Jakob, le Système canadien de surveillance de la maladie de Creutzfeldt-Jakob a démontré sa capacité à détecter, étudier et gérer des événements rares, comme les quatre cas recensés de $\mathrm{MCJ}$ i et les deux cas de vMCJ. Nos résultats indiquent, par conséquent, qu'un environnement axé sur la collaboration a été établi pour assurer l'efficacité de la surveillance épidémiologique prospective nationale de la maladie de Creutzfeldt-Jakob au Canada.

Il est important que les professionnels de la santé restent alertes à la possibilité d'une maladie de CreutzfeldtJakob chez leurs patients. De même, il est important que les professionnels de santé publique utilisent les données de surveillance et leurs résultats épidémiologiques pour évaluer et atténuer les risques de santé publique associés à l'occurrence continue, éventuellement sous-constatée et potentiellement en hausse, des formes classiques de la maladie de Creutzfeldt-Jakob. Enfin, il subsiste des incertitudes concernant l'épidémiologie de la $\mathrm{VMCJ}$ et des nouvelles maladies zoonotiques à prion possibles, qui justifient de rester vigilants aux nouveaux événements ou tendances de la maladie de Creutzfeldt-Jakob $(37,38)$.

\section{Remerciements}

Les auteurs tiennent à remercier sincèrement les nombreux professionnels de la santé, établissements de soins de santé, fournisseurs de service et, surtout, les patients touchés et leur famille; ce travail n'aurait pas été possible sans leur généreuse collaboration. Ils remercient aussi tout particulièrement les membres du personnel de la section des maladies à prion du Laboratoire national de microbiologie, des laboratoires nationaux de santé publique, de l'Agence de la santé publique du Canada et des Services de laboratoire de neuropathologie de I'Université d'Ottawa, pour leur compétence et leur dévouement à fournir des services de diagnostic en laboratoire d'excellente qualité. Les chiffres de la population canadienne et les données démographiques utilisés dans le présent rapport ont été adaptés de Statistique Canada; cette mention ne fait en aucun cas office d'approbation par Statistique Canada de la présente publication.

\section{Conflit d'intérêts}

Aucun.

\section{Financement}

Le Système canadien de surveillance de la maladie de Creutzfeldt-Jakob est financé dans le cadre du Programme des maladies à prion de l'Agence de la santé publique du Canada. 


\section{Références}

(1) Prusiner SB. Prions. Proc Natl Acad Sci U S A. 1998 Nov 10;95(23):13363-83.

(2) Kraus A, Groveman BR, Caughey B. Prions and the potential transmissibility of protein misfolding diseases. Annu Rev Microbiol. 2013;67:543-64.

(3) Cashman NR. Propagated protein misfolding: New opportunities for therapeutics, new public health risk. CCDR. 2015;41(8):196-200.

(4) Belay ED, Schonberger LB. The public health impact of prion diseases. Ann Rev Public Health. 2005;26:191-212.

(5) Ladogana A, Puopolo M, Croes EA, Budka H, Jarius C, Collins S, et al. Mortality from Creutzfeldt-Jakob disease and related disorders in Europe, Australia, and Canada. Neurology. 2005 May 10;64(9):1586-91.

(6) Kovacs GG, Puopolo M, Ladogana A, Pocchiari M, Budka H, van Duijn C, et al. Genetic prion disease: the EUROCJD experience. Hum Genet. 2005 Nov;118(2):166-74.

(7) Brown P, Brandel JP, Sato T, Nakamura Y, MacKenzie J, Will RG, et al. latrogenic Creutzfeldt-Jakob disease, final assessment. Emerg Infect Dis. 2012 Jun;18(6):901-7.

(8) de Pedro-Cuesta J, Mahillo-Fernandez I, Rabano A, Calero M, Cruz M, Siden A, et al. Nosocomial transmission of sporadic Creutzfeldt-Jakob disease: Results from a risk-based assessment of surgical interventions. J Neurol Neurosurg Psychiatry. 2011 Feb;82(2):204-12.

(9) de Pedro-Cuesta J, Mahillo-Fernandez I, Calero M, Rabano A, Cruz M, Siden A, et al. Towards an age-dependent transmission model of acquired and sporadic Creutzfeldt-Jakob disease PLoS One. 2014 Oct 3;9(10):e109412.

(10) Coulthart MB, Cashman NR. Variant Creutzfeldt-Jakob disease: A summary of current scientific knowledge in relation to public health. CMAJ. 2001 Jul 10;165(1):51-8.

(11) Will RG, Ironside JW, Zeidler M, Cousens SN, Estibeiro K, Alperovitch A, et al. A new variant of Creutzfeldt-Jakob disease in the UK Lancet. 1996 Apr 6;347(9006):921-5.

(12) Diack AB, Head MW, McCutcheon S, Boyle A, Knight R, Ironside JW, et al. Variant CJD. 18 years of research and surveillance. Prion. 2014;8(4):286-95.

(13) Jansen GH, Voll CL, Robinson CA, Gervais R, Sutcliffe T, Bergeron C, et al. First case of variant Creutzfeldt-Jakob disease in Canada. CCDR. 2003 Jul 1;29(13):117-20.

(14) Public Health Agency of Canada (PHAC). Variant Creutzfeldt-Jakob disease in a Canadian resident. CCDR Weekly. Inf Dis News Brief. 2011;4(10).

(15) Hewitt PE, Llewelyn CA, Mackenzie J, Will RG. Creutzfeldt-Jakob disease and blood transfusion: results of the UK Transfusion Medicine Epidemiological Review study. Vox Sang. 2006 Oct;91(3):221-30.

(16) Brown P. Creutzfeldt-Jakob disease: Reflections on the risk from blood product therapy. Haemophilia. 2007 Dec;13 Suppl 5:33-40.

(17) Peden A, McCardle L, Head MW, Love S, Ward HJ, Cousens SN, et al. Variant CJD infection in the spleen of a neurologically asymptomatic UK adult patient with haemophilia. Haemophilia. 2010 Mar;16(2):296-304.

(18) World Health Organization (WHO). WHO manual for surveillance of human transmissible spongiform encephalopathies including variant Creutzfeldt-Jakob disease. Geneva: WHO; 2003. http://whqlibdoc.who.int/publications/2003/9241545887.pdf

(19) Krever H. Commission of Inquiry on the Blood System in Canada: Final Report. Ottawa: Government of Canada; 1997.

(20) Ricketts MN, Cashman NR, Stratton EE, ElSaadany S. Is Creutzfeldt-Jakob disease transmitted in blood? Emerg Infect Dis. 1997 Apr-Jun;3(2):155-63.

(21) Coulthart MB, Stratton EE, Ricketts MN. Surveillance of Creutzfeldt-Jakob disease in Canada. Can J Infect Dis. 1997;8(5):241-4

(22) Murray K. Creutzfeldt-Jakob disease mimics, or how to sort out the subacute encephalopathy patient. Pract Neurol. $2011 \mathrm{Feb} ; 11(1): 19-28$.

(23) Public Health Agency of Canada. Case definitions for communicable diseases under national surveillance: 2009. CCDR. 2009;35(S2):1-128.

(24) CANSIM: Canadian Socioeconomic Information Management database, Table 051-0001 (reference date 2014-0924), Table 97-551-X2006008 (reference date 2007-07-17).

www.statcan.gc.ca

(25) Porta M. A Dictionary of Epidemiology. 4th ed. New York: Oxford University Press; 2008.

(26) Conover WJ. Practical Nonparametric Statistics. 3rd ed. New York: Wiley; 1999.

(27) Info Source: Sources of federal government and employee information. Record number PHAC 008 180, Treasury Board Secretariat registration number 009940, Bank number PHAC PPU 286. 2014 Sep 3.

http://www.phac-aspc.gc.ca/about_apropos/atip-aiprp/info-source-eng.php

(28) Masters CL, Harris JO, Gajdusek DC, Gibbs CJ, Jr, Bernoulli C, Asher DM. Creutzfeldt-Jakob disease: Patterns of worldwide occurrence and the significance of familial and sporadic clustering. Ann Neurol. 1979 Feb;5(2):177-88.

(29) Will RG, Matthews WB, Smith PG, Hudson C. A retrospective study of Creutzfeldt-Jakob disease in England and Wales 1970-1979. II: Epidemiology. J Neurol Neurosurg Psychiatry. 1986 Jul;49(7):749-55. 
(30) Brown P, Cathala F, Raubertas RF, Gajdusek DC, Castaigne P. The epidemiology of Creutzfeldt-Jakob disease: Conclusion of a 15-year investigation in France and review of the world literature. Neurology. 1987 Jun;37(6):895-904.

(31) Cousens SN, Zeidler M, Esmonde TF, De Silva R, Wilesmith JW, Smith PG, et al. Sporadic Creutzfeldt-Jakob disease in the United Kingdom: Analysis of epidemiological surveillance data for 1970-96. BMJ. 1997 Aug 16;315(7105):389-95.

(32) Stoeck K, Hess K, Amsler L, Eckert T, Zimmermann D, Aguzzi A, et al. Heightened incidence of sporadic Creutzfeldt-Jakob disease is associated with a shift in clinicopathological profiles. J Neurol. 2008 Oct;255(10):1464-72.

(33) Ruegger J, Stoeck K, Amsler L, Blaettler T, Zwahlen M, Aguzzi A, et al. A case-control study of sporadic Creutzfeldt-Jakob disease in Switzerland: Analysis of potential risk factors with regard to an increased CJD incidence in the years 2001-2004. BMC Public Health. 2009 Jan 14;9:18. doi: 10.1186/1471-2458-9-18.

(34) Klug GM, Wand H, Simpson M, Boyd A, Law M, Masters CL, et al. Intensity of human prion disease surveillance predicts observed disease incidence. J Neurol Neurosurg Psychiatry. 2013 Dec;84(12):1372-7.

(35) Stratton E, Ricketts MN, Gully PR. The epidemiology of Creutzfeldt-Jakob disease in Canada: A review of mortality data. Emerg Infect Dis. 1997 Jan-Mar;3(1):63-4.

(36) Elsaadany S, Semenciw R, Ricketts M, Mao Y, Giulivi A. Epidemiological study of Creutzfeldt-Jakob disease death certificates in Canada, 1979-2001. Neuroepidemiology. 2005;24(1-2):15-21.

(37) Saunders SE, Bartelt-Hunt SL, Bartz JC. Occurrence, transmission, and zoonotic potential of chronic wasting disease. Emerg Infect Dis. 2012 Mar;18(3):369-76.

(38) Gill ON, Spencer Y, Richard-Loendt A, Kelly C, Dabaghian R, Boyes L, et al. Prevalent abnormal prion protein in human appendixes after bovine spongiform encephalopathy epizootic: Large scale survey. BMJ. 2013 Oct 15;347:f5675. 